\title{
Finite-Time Estimation of Multiple Exponentially-Damped Sinusoidal Signals: A Kernel-based Approach *
}

\author{
Boli Chen ${ }^{\mathrm{a}}$, Peng Li ${ }^{\mathrm{a}}$, Gilberto Pin ${ }^{\mathrm{b}}$, Giuseppe Fedele ${ }^{\mathrm{c}}$, Thomas Parisini ${ }^{\mathrm{a}, \mathrm{d}, \mathrm{e}}$ \\ ${ }^{a}$ Dept. of Electrical and Electronic Engineering, Imperial College London, UK \\ ${ }^{\mathrm{b}}$ Electrolux Italia S.p.A, Italy \\ ${ }^{\mathrm{c}}$ DIMES, University of Calabria, Rende (CS), Italy \\ ${ }^{\mathrm{d}}$ Dept. of Engineering and Architecture, University of Trieste, Italy \\ ${ }^{\mathrm{e}}$ KIOS Research and Innovation Centre of Excellence, University of Cyprus
}

\begin{abstract}
The problem of estimating the parameters of biased and exponentially-damped multi-sinusoidal signals is addressed in this paper by a finite-time identification scheme based on Volterra integral operators. These parameters are the amplitudes, frequencies, initial phase angles, damping factors and the offset. The proposed strategy entails the design of a new kind of kernel function that, compared to existing ones, allows for the identification of the initial conditions of the signal-generator system. The worst-case behaviour of the proposed algorithm in the presence of bounded additive disturbances is fully characterized by Input-to-State Stability arguments. Numerical examples including the comparisons with some existing tools are reported to show the effectiveness of the proposed methodology.
\end{abstract}

\section{Introduction}

Exponentially Damped Sinusoidal (EDS) signals can be observed in a wide range of natural phenomena, like the propagation of acoustic waves and may as well characterize the behaviour of artificial systems, occurring for instance as a consequence of the complex interaction between power systems components. The parametric estimation of EDS signals is crucial as the sinusoidal parameters describe the qualitative behaviour of the associated system. For example, oscillations with time-varying amplitude in power systems might be the precursor of instabilities due to equipment malfunctions or other faults. In this context, precise and fast identification of these oscillations has drawn considerable research activities.

The Prony's method and its many variants represent

\footnotetext{
‡ This work has been supported by the European Union's Horizon 2020 Research and Innovation Program under the grant agreement No. 739551 (KIOS CoE).

Email addresses: boli.chen10@imperial.ac.uk (Boli Chen), peng.li13@imperial.ac.uk (Peng Li), gilbertopin@alice.it (Gilberto Pin), giuseppe.fedele@unical.it (Giuseppe Fedele), t.parisini@gmail.com (Thomas Parisini ).
}

the most traditional techniques, designed for estimating frequency components of damped sinusoids in a complex form $[1,2]$. However, these methods are sensitive to the measurement noise. In this context, several highresolution alternatives have been proposed in the literature, such as the Yule-Walker algorithm [3], matrix pencil methods [4] and algorithms based on the Hilbert Transformation [5]. In the context of continuous-time estimation, a wide variety of techniques have been proposed to address the estimation problem of non-fading sinusoidal signals with asymptotic stability (see, for example, [6-10], and the references cited therein). Some of these methods have also been extended to address the EDS estimation problem, for example, the estimators based on the internal model principle [13] and the second-order generalized integrator [14]. A further important class of algorithms used in the context of EDS estimation is represented by the so-named algebraic identification methods. They are characterized by very rapid detection of the parameters (non-asymptotic and finitetime convergence), which is a crucial feature as the input signal may gradually vanish due to the positive damping or the negative damping may lead an instability issue $[11,12]$. Compared to the Prony's methods, algebraic methods based on differential algebra and operational calculus are more robust against the measurement noise. 
As shown for the first time in [15], the Volterra integral operator turns out to be an effective tool for developing finite-time estimation algorithms. For example, the estimation problem of a single non-fading sinusoidal signal is addressed in [16]. Resorting to the Volterra integral operator with suitably designed kernel functions, the method can achieve finite-time convergence. In a more recent work [17], the estimator proposed in [16] has been extended to an enhanced algorithm that is able to estimate parameters of a biased and damped sinusoidal signal.

Based on some preliminary results presented in [18], this paper deals with a finite-time estimation scheme for estimating characteristics of multiple biased and damped sinusoidal signals. With inspiration from the idea devised in [15], the proposed methodology employs Volterra operators with original kernel functions, named Bivariate Linear Non-asymptotic Kernels (BL-NK). Compared to the previous kernels proposed by the authors in [16] and [17], the presented ones preserve the dependency on the initial conditions. As such, the initial amplitudes and the phases of the sinusoidal components can be determined in addition to the frequencies. The robustness of the devised method with respect to the bounded additive measurement perturbation is characterized by Input-toState Stability (ISS) tools. Finally, the performance of the proposed algorithm is evaluated by various simulation examples including comparisons with some existing deadbeat techniques.

This paper is organized as follows: the problem is formulated in Section 2 with some basic definitions. In Section 3 , the kernel-based estimation scheme is proposed. Stability and robustness analysis with respect to measurement perturbations is carried out in Section 4. Extensive simulation results are provided in Section 5, while Section 6 draws some concluding remarks.

\section{Problem statement and preliminaries}

Consider the following perturbed signal made up of a finite number of damped sinusoids and a constant bias

$$
y(t)=\sum_{i=1}^{n} A_{i} e^{\rho_{i} t} \sin \left(\omega_{i} t+\phi_{i}\right)+A_{0}
$$

with known $n \geq 1$ and $4 n+1$ unknown parameters including amplitudes $A_{i} \in \mathbb{R}_{>0}$, frequencies $\omega_{i} \in \mathbb{R}_{>0}$, initial phase angles $\phi_{i} \in[0,2 \pi]$, damping factors $\rho_{i} \in \mathbb{R}$ and the offset $A_{0}$. Moreover, it is also assumed that the frequencies verify the relationship $\omega_{i} \neq \omega_{j}$ for $i \neq j$.

It is worth noting that (1) can be generated by the follow- ing observable autonomous marginally-stable system:

$$
\left\{\begin{array}{l}
\dot{\mathbf{w}}(t)=\mathbf{A}_{w} \mathbf{w}(t) \\
y(t)=\mathbf{c}_{w}^{\top} \mathbf{w}(t)
\end{array}\right.
$$

where $\quad \mathbf{w}(t) \triangleq\left[\begin{array}{llll}w_{0}(t) \ldots w_{r}(t) \ldots w_{2 n}(t) & \ldots\end{array}\right]^{\top} \in \mathbb{R}^{2 n+1}$, and the initial condition

$$
\begin{aligned}
& w_{2 i-2}(0)=A_{i} \sin \phi_{i}, w_{2 i-1}(0)=A_{i} \omega_{i} \cos \phi_{i}, \\
& w_{2 n}(0)=A_{0}, \forall i \in\{1, \ldots, n\} .
\end{aligned}
$$

The system matrices are given as follows

$$
\mathbf{A}_{w}=\left[\begin{array}{ccccc}
\mathbf{J}_{1} & 0 & 0 & \cdots & 0 \\
0 & \ddots & \ddots & \ddots & 0 \\
\vdots & \ddots & 0 & \mathbf{J}_{n} & 0 \\
0 & \cdots & 0 & 0 & 0
\end{array}\right], \mathbf{c}_{w}^{\top}=\left[\begin{array}{c}
c_{1} \\
\vdots \\
c_{n} \\
1
\end{array}\right]^{\top}
$$

with

$$
\mathbf{J}_{i}=\left[\begin{array}{cc}
\rho_{i} & 1 \\
-\omega_{i}^{2} & \rho_{i}
\end{array}\right], c_{i}^{\top}=\left[\begin{array}{ll}
1 & 0
\end{array}\right] .
$$

The associated characteristic polynomial, having $2 n$ complex roots $\rho_{i} \pm j \omega_{i}, i=1,2, \ldots, n$ occurring in complex-conjugate pairs and one root at zero, is given by

$$
P(s)=s^{2 n+1}+\alpha_{2 n} s^{2 n}+\cdots+\alpha_{2} s^{2}+\alpha_{1} s,
$$

where $s$ is Laplace variable, $\left(\alpha_{1}, \alpha_{2}, \cdots, \alpha_{2 n}\right)$ are the coefficients of the characteristic polynomial, determined by the unknown frequencies $\omega_{i}$ and damping factors $\rho_{i}, i=1,2, \cdots, n$.

Being (2) observable, the state vector $\mathbf{w}(t)$ admits a linear transformation of coordinates $\mathbf{z}(t)=\mathbf{T w}(t)$, such that the signal generator of $y(t)$ can be rewritten in an observer canonical form. Let

$$
\mathbf{z}(t) \triangleq\left[\begin{array}{llllll}
z_{0}(t) & z_{1}(t) & \ldots & z_{r}(t) & \ldots & z_{2 n}(t)
\end{array}\right]^{\top} \in \mathbb{R}^{2 n+1},
$$

the canonical system evolving from the unknown initial state $\mathbf{z}(0)=\mathbf{T w}(0)$, is given as follows:

$$
\left\{\begin{array}{l}
\dot{\mathbf{z}}(t)=\mathbf{A}_{z} \mathbf{z}(t), \\
y(t)=\mathbf{c}_{z}^{\top} \mathbf{z}(t), \quad t \in \mathbb{R}_{\geq 0},
\end{array}\right.
$$

where $\mathbf{A}_{z}=\mathbf{T} \mathbf{A}_{w} \mathbf{T}^{-1}, \mathbf{c}_{z}^{\top}=\mathbf{c}_{w}^{\top} \mathbf{T}^{-1}$ are given by

$$
\mathbf{A}_{z}=\left[\begin{array}{ccccc}
-\alpha_{2 n} & 1 & 0 & \cdots & 0 \\
\vdots & 0 & \ddots & \ddots & 0 \\
-\alpha_{1} & \vdots & \ddots & \ddots & 1 \\
0 & 0 & \cdots & 0 & 0
\end{array}\right], \mathbf{c}_{z}^{\top}=\left[\begin{array}{c}
1 \\
0 \\
\vdots \\
0
\end{array}\right]^{\top}
$$


In the following, a deadbeat algorithm is introduced to address the identification of the unknown system parameters $\alpha_{i}$ and the initial conditions $\mathbf{z}(0)$. Thereby the frequencies and damping factors are respectively determined as the imaginary and real parts of the zeros of the characteristic polynomial $P(s)$. The amplitudes and initial phase shifts are estimated by (3) thanks to the estimated frequencies and damping factors.

Some basic concepts concerning the Volterra integral are recalled in the following for the reader's convenience. The reader is referred to $[15,19]$ for a deeper insight on this topic.

Letting $x(t) \in \mathbb{R}, \forall t \geq 0$ be an $i$-th order differentiable signal, in this paper we denote by $x^{(i)}$ the $i$-th order derivative signal. Moreover, given a kernel function $K(\cdot, \cdot)$ in two variables, its $i$-th order partial derivative with respect to the second argument will be denoted as $K^{(i)}(t, \tau), i \in \mathbb{Z}_{\geq 0}$.

Consider a Volterra integral operator induced by the kernel function $K(\cdot, \cdot)$

$$
\left[V_{K} x\right](t) \triangleq \int_{0}^{t} K(t, \tau) x(\tau) d \tau, \quad t \in \mathbb{R}_{\geq 0}
$$

For the sake of practical implementability, we use the following dynamic system to compute the transformed signal $\left[V_{K} x\right](t)$, for $t \geq 0$ :

$$
\left\{\begin{aligned}
\xi^{(1)}(t) & =K(t, t) x(t)+\int_{0}^{t}\left(\frac{\partial}{\partial t} K(t, \tau)\right) x(\tau) d \tau \\
{\left[V_{K} x\right](t) } & =\xi(t)
\end{aligned}\right.
$$

where $\xi(0)=\xi^{(1)}(0)=0$.

The following result is useful in dealing with the application of Volterra operators to the derivatives of a signal.

Lemma 2.1 [15] For a given $i \geq 0$, consider a signal $x(\cdot) \in \mathcal{L}^{2}\left(\mathbb{R}_{\geq 0}\right)$ that admits an $i$-th weak derivative in $\mathbb{R}_{\geq 0}$ and a kernel function $K(\cdot, \cdot) \in \mathcal{H S}$, admitting the $i$-th derivative (in the conventional sense) with respect to the second argument. Then, it holds that:

$$
\begin{aligned}
& {\left[V_{K} x^{(i)}\right](t)=\sum_{j=0}^{i-1}(-1)^{i-j-1} x^{(j)}(t) K^{(i-j-1)}(t, t)} \\
& +\sum_{j=0}^{i-1}(-1)^{i-j} x^{(j)}(0) K^{(i-j-1)}(t, 0)+(-1)^{i}\left[V_{K^{(i)}} x\right](t)
\end{aligned}
$$

that is, the function $\left[V_{K} x^{(i)}\right](\cdot)$ can be obtained by the lower-order derivatives $x(\cdot), x^{(1)}(\cdot), \ldots, x^{(i-1)}(\cdot)$.
The properties of the Volterra operator $V_{K}$ depend significantly on the characteristics of the kernel function, which determine the application domain of the specific operator $[15,20]$. In this work, we introduce a new family of kernel functions, BL-NK, that allow estimating simultaneously the parameters of a linear system as well as its initial state.

Definition 2.1 (BL-NK) If a function $K(\cdot, \cdot) \in \mathcal{H S}$ which is at least $(i-1)$-th order differentiable with respect to the second argument, verifies the conditions

$$
K^{(j)}(t, t)=0, K^{(j)}(t, 0)>0, \forall t>0,
$$

$\forall j \in\{0,1, \ldots, i-1\}$, then, it is called an $i$-th order $B i$ variate Linear Non-asymptotic Kernel.

The following instance of BL-NK (fulfilling (10)) will be considered throughout the paper:

$$
K(t, \tau)=e^{-\beta(t-\tau)}\left(1-e^{-\beta(t-\tau)}\right)^{N}
$$

with the parameter $\beta \in \mathbb{R}_{>0}$ set arbitrarily.

\section{Finite-time amplitude, frequency and phase estimation}

For the sake of further discussion, it is worth to introduce the differential-constraint model of (5):

$$
\left\{\begin{aligned}
y^{(2 n+1)}(t) & =\sum_{i=1}^{2 n}-\alpha_{i} y^{(i)}(t), \forall t \in \mathbb{R}_{\geq 0} \\
y^{(i)}(0) & =y_{0}^{(i)}, i \in\{0, \ldots, 2 n+1\}
\end{aligned}\right.
$$

where $y_{0}^{(i)}, i \in\{0, \ldots, 2 n+1\}$ represent the unknown initial conditions. Notably, the state-variables of the observer canonical realization (5) can be expressed as a linear combination of the output derivatives:

$$
z_{r}(t)=y^{(r)}(t)+\sum_{j=0}^{r-1} \alpha_{2 n-r+j+1} y^{(j)}(t), r \in\{0,1, \cdots, 2 n\}
$$

where we have used the convention $\sum_{j=0}^{k}\{\cdot\}=0, \forall k<0$.

Assuming that $K(\cdot, \cdot)$ is an at least $(2 n+1)$-th order Bivariate Causal kernel function satisfying condition (10), thanks to Lemma 2.1, it is immediately to show that

$$
\begin{array}{r}
{\left[V_{K} y^{(i)}\right](t)=\sum_{j=0}^{i-1}(-1)^{i-j} y^{(j)}(0) K^{(i-j-1)}(t, 0)} \\
+(-1)^{i}\left[V_{K^{(i)}} y\right](t)
\end{array}
$$


for all $i \in\{0, \cdots, 2 n+1\}$. Therefore, by applying the operator $V_{K}$ to both sides of (12), recall (14) it holds that

$$
\begin{array}{r}
(-1)^{2 n}\left[V_{K^{(2 n+1)}} y\right](t)=-y^{(2 n)}(0) K(t, 0) \\
-\sum_{j=0}^{2 n-1}(-1)^{2 n-j} y^{(j)}(0) K^{(2 n-j)}(t, 0) \\
+\sum_{i=1}^{2 n} \alpha_{i}\left(\sum_{j=0}^{i-1}(-1)^{i-j} y^{(j)}(0) K^{(i-j-1)}(t, 0)\right. \\
\left.+(-1)^{i}\left[V_{K^{(i)}} y\right](t)\right)
\end{array}
$$

By applying (13) and after some cumbersome algebra, we get

$$
\begin{aligned}
{\left[V_{K^{(2 n+1)}} y\right](t)=} & \sum_{i=0}^{2 n-1} \alpha_{i}(-1)^{i+1}\left[V_{K^{(i+1)}} y\right](t) \\
& -\sum_{r=0}^{2 n}(-1)^{2 n-r} K^{(2 n-r)}(t, 0) z_{r}(0)
\end{aligned}
$$

which can be written in compact form

$$
\left[V_{K^{(2 n+1)}} y\right](t)=\sum_{i=0}^{2 n-1} \alpha_{i}(-1)^{i+1}\left[V_{K^{(i+1)}} y\right](t)+\sum_{r=0}^{2 n} \gamma_{r}(t) z_{r}(0)
$$

with $\gamma_{r}(t)=K^{(2 n-r)}(t, 0)(-1)^{2 n-r+1}$.

Noting that the right hand side of (15) is linear with respect to the parameters $\alpha_{i}$ and the initial state $z_{r}(0)$, it can be recast in the following vector form

$$
\left[V_{K^{(2 n+1)}} y\right](t)=\boldsymbol{\nu}(t)^{\top} \boldsymbol{\theta}
$$

where $\boldsymbol{\theta}=\left[\alpha_{1}, \alpha_{2}, \ldots, \alpha_{2 n}, z_{0}(0), z_{1}(0), \ldots, z_{2 n}(0)\right]^{\top} \in \mathbb{R}^{3 n}$. is an extended parameter vector that contains, besides model parameters, also the initial conditions of output derivatives, while the regressors vector $\boldsymbol{\nu}(t)$ is given by

$$
\begin{array}{r}
\boldsymbol{\nu}(t)=\left[-\left[V_{K^{(1)}} y\right](t),\left[V_{K^{(2)}} y\right](t), \ldots,\left[V_{K^{(2 n)}} y\right](t),\right. \\
\left.\gamma_{0}(t), \gamma_{1}(t), \ldots, \gamma_{2 n}(t)\right]^{\top} .
\end{array}
$$

For the sake of the further discussion, it is worth to point out that $\boldsymbol{\nu}(t)$ is not only made up of known timedependent functions $\gamma_{0}(t), \gamma_{1}(t), \ldots, \gamma_{2 n}(t)$ but also of signals obtained by processing $y(t)$ through the Volterra operators. Moreover, to solve (16), we also need to calculate the signal $\left[V_{K^{(2 n+1)}} y\right](t)$, which involves processing the output by a further Volterra operator. In the following lines we will show how these signals can be obtained, deriving a state-space realization for the
Volterra-processing mechanism. To this end, let us introduce a vector containing all the transformed signals:

$$
\begin{aligned}
\mathbf{z}_{a}(t) \triangleq\left[-\left[V_{K^{(1)}} y\right](t),[\right. & \left.V_{K^{(2)}} y\right](t), \ldots, \\
& {\left.\left[V_{K^{(2 n)}} y\right](t),\left[V_{K^{(2 n+1)}} y\right](t)\right]^{\top} }
\end{aligned}
$$

We will show that $\mathbf{z}_{a}(t)$ can be computed as the output of an internally stable LTI system.

Consider a BL-NK in the form of (11) with $N=2 n+2$ :

$$
K(t, \tau)=e^{-\beta(t-\tau)}\left(1-e^{-\beta(t-\tau)}\right)^{2 n+2}
$$

where $\beta \in \mathbb{R}_{>0}$ is chosen by the designers. The $i$-th derivative of the designed kernel with respect to the second argument can be expressed as:

$$
K^{(i)}(t, \tau)=\sum_{j=1}^{2 n+3} e^{-j \beta t} f_{i, j}(\tau), \forall i \in\{1,2, \ldots, 2 n+1\}
$$

where $f_{i, j}(\tau)=\left(\begin{array}{c}2 n+2 \\ j-1\end{array}\right)(-1)^{j-1}(j \beta)^{i} e^{j \beta \tau}$. are univariate functions of $\tau$. Let $K_{i, j}(t, \tau) \triangleq e^{-j \beta t} f_{i, j}(\tau)$, then we have

$$
\frac{\partial}{\partial t} K_{i, j}(t, \tau)=-j \beta e^{-j \beta t} f_{i, j}(\tau) .
$$

Moreover, by the linearity of the Volterra operator, it follows that $\left[V_{K^{(i)}} y\right](t)=\sum_{j=1}^{2 n+3}\left[V_{K_{i, j}} y\right](t)$. Defining the internal state vector $\boldsymbol{\xi}(t) \in \mathbb{R}^{(2 n+1) \times(2 n+3)}$

$$
\boldsymbol{\xi}(t) \triangleq\left[\xi_{1,1}(t), \ldots, \xi_{1,2 n+3}, \xi_{2,1}(t), \ldots, \xi_{2 n+1,2 n+3}\right]^{\top},
$$

with $\xi_{i, j}(t) \triangleq\left[V_{K_{i, j}} y\right](t)$. Then, owing to (8), the augmented signal vector $\mathbf{z}_{a}(t)$ can be computed by the following stable LTI system:

$$
\left\{\begin{aligned}
\boldsymbol{\xi}^{(1)}(t) & =\mathbf{G}_{\xi} \boldsymbol{\xi}(t)+\mathbf{E} y(t) \\
\mathbf{z}_{a}(t) & =\mathbf{H} \boldsymbol{\xi}(t)
\end{aligned}\right.
$$

with $\boldsymbol{\xi}(0)=0 \in \mathbb{R}^{(2 n+1)(2 n+3)}$ and where $\mathbf{G}_{\xi}$ is a diagonal, time invariant and Hurwitz matrix, defined by $\mathbf{G}_{\xi}=\operatorname{blockdiag}[\mathbf{G}, \ldots, \mathbf{G}]$, with $\mathbf{G}=$ $\operatorname{diag}(-\beta,-2 \beta, \ldots,-(2 n+3) \beta)$, and $\mathbf{H}$ is defined by $\mathbf{H}=\operatorname{blockdiag}\left[\mathbf{1}^{\top}, \ldots, \mathbf{1}^{\top}\right]$, with $\mathbf{1}^{\top}$ denoting a row vector of ones with $2 n+3$ elements. Finally, due to the fact that the functions $K_{i, j}(t, \tau)$ evaluated for $\tau=t$ are constants:

$$
K_{i, j}(t, t)=\lambda_{i, j} \triangleq(-1)^{i+j}\left(\begin{array}{c}
2 n+2 \\
j-1
\end{array}\right)(j \beta)^{i} .
$$


$\mathbf{E}=\left[\mathbf{E}_{1}^{\top}, \ldots, \mathbf{E}_{2 n+1}^{\top}\right]^{\top}$ is a constant vector given by: $\mathbf{E}_{i}=$ $\left[\lambda_{i, 1}, \lambda_{i, 2}, \ldots, \lambda_{i, 2 n+3}\right]^{\top}$. In view of (16), conventional augmentation tools used in system's identification can be employed to form a well-posed algebraic system. Let us multiple $\boldsymbol{\nu}(t)$ on both sides of (16), leading to:

$$
\mathbf{S}(t)=\mathbf{R}(t) \boldsymbol{\theta}
$$

where $\mathbf{S}(t) \triangleq \boldsymbol{\nu}(t)\left[V_{K^{(2 n+1)}} y\right](t) \in \mathbb{R}^{(4 n+1)}, \mathbf{R}(t) \triangleq$ $\boldsymbol{\nu}(t) \boldsymbol{\nu}^{\top}(t) \in \mathbb{R}^{(4 n+1) \times(4 n+1)}$. To avoid singularity of the instantaneous auto-covariance matrix $\mathbf{R}(t)$, we apply to both sides of (21) a first order linear filter $G_{f}(s)=$ $1 /(s+g)$ with zero initial conditions. Then it holds that

$$
\mathscr{L}^{-1}\left\{G_{f}(s) \mathbf{S}(s)\right\}(t)=\mathscr{L}^{-1}\left\{G_{f}(s) \mathbf{R}(\mathbf{s})\right\}(t) \theta .
$$

Consider $\mathbf{S}_{f}(t) \triangleq \mathscr{L}^{-1}\left\{G_{f}(s) \mathbf{S}(s)\right\}(t)$ and $\mathbf{R}_{f}(t) \triangleq$ $\mathscr{L}^{-1}\left\{G_{f}(s) \mathbf{R}(\mathbf{s})\right\}(t)$, it follows that

$$
\left\{\begin{array}{l}
\dot{\mathbf{S}}_{f}(t)=-g \mathbf{S}_{f}(t)+\mathbf{S}(t) \\
\dot{\mathbf{R}}_{f}(t)=-g \mathbf{R}_{f}(t)+\mathbf{R}(t)
\end{array}\right.
$$

where $\mathbf{S}_{f}(0)=0 \in \mathbb{R}^{(4 n+1)}, \mathbf{R}_{f}(0)=0 \in \mathbb{R}^{(4 n+1) \times(4 n+1)}$. It is clear that the filtering operator is equivalent of using Volterra operator with a kernel $G_{f}(t, \tau)=e^{-g(t-\tau)}$.

For the sake of further analysis, let us expand $\gamma_{r}(t), r=$ $0,1, \ldots, 2 n$ that is contained in the regressor $\boldsymbol{\nu}(t)$ :

$$
\gamma_{r}(t)=(-1)^{2 n-r+1} \sum_{j=1}^{2 n+3} e^{-j \beta t} f_{2 n-r, j}(0)
$$

where $f_{2 n-r, j}(0)=(-1)^{j-1}\left(\begin{array}{c}2 n+2 \\ j-1\end{array}\right)(j \beta)^{2 n-r}, \forall j \quad \in$ $\{1, \ldots, 2 n+3\}, r \in\{0, \ldots, 2 n\}$ are constants. Hence, let

$$
\mathcal{F}_{r, j}=(-1)^{2 n-r+j}\left(\begin{array}{c}
2 n+2 \\
j-1
\end{array}\right)(j \beta)^{2 n-r},
$$

$\gamma_{r}(t)$ can be represented as the sum of exponential functions $\gamma_{r}(t)=\sum_{j=1}^{2 n+3} e^{-j \beta t} \mathcal{F}_{r, j}$ which decay to zero as $t \rightarrow \infty$. The following technical result characterizes a specialized persistency of excitation (PE) on signal $\boldsymbol{\nu}(t)$ that is needed to prove the convergence of the devised estimation algorithm.

Lemma 3.1 (Finite-time persistency of excitation) Given the multi-EDS measurement $y(t)$ (see (1)) and the designed kernel (18), there exist some $\in \in \mathbb{R}_{>0}, t_{\epsilon} \in \mathbb{R}_{>0}$ and $T \in \mathbb{R}_{>0}$ such that

$$
\int_{t-t_{\epsilon}}^{t} \boldsymbol{\nu}(\tau) \boldsymbol{\nu}^{\top}(\tau) d \tau \geq \epsilon \mathbf{I}, \forall t \in\left[t_{\epsilon}, t_{\epsilon}+T\right] .
$$

Proof. In view of (17), we can split $\boldsymbol{\nu}(t)$ into two vector signals $\boldsymbol{\nu}_{1}(t) \in \mathbb{R}^{2 n}$ and $\boldsymbol{\nu}_{2}(t) \in \mathbb{R}^{2 n+1}$, such that

$$
\mathscr{L}\left\{\boldsymbol{\nu}_{1}(t)\right\}=\mathbf{G}_{1}(s) \mathscr{L}\{y(t)\}, \boldsymbol{\nu}_{2}(t)=\mathbf{G}_{2} \boldsymbol{\psi}_{2}(t) .
$$

where $\mathbf{G}_{1}(s)=\left[\kappa_{1}(s) \kappa_{2}(s) \cdots \kappa_{2 n}(s)\right]^{\top} \in \mathbb{C}^{2 n}$ with

$$
\begin{aligned}
\kappa_{i}(s) \triangleq & \sum_{j=1}^{2 n+3} \frac{\lambda_{i, j}}{s+j \beta}, i=1,2, \ldots, 2 n, \text { and } \\
\boldsymbol{\psi}_{2}(t) \triangleq & {\left[\begin{array}{cccc}
e^{-\beta t} & e^{-2 \beta t} & \cdots & e^{-(2 n+3) \beta t}
\end{array}\right]^{\top}, } \\
\mathbf{G}_{2}= & {\left[\begin{array}{cccc}
\mathcal{F}_{0,1} & \mathcal{F}_{0,2} & \cdots & \mathcal{F}_{0,2 n+3} \\
\mathcal{F}_{1,1} & \mathcal{F}_{1,2} & \cdots & \mathcal{F}_{1,2 n+3} \\
\vdots & \cdots & \ddots & \vdots \\
\mathcal{F}_{2 n, 1} & \mathcal{F}_{2 n, 2} & \cdots & \mathcal{F}_{2 n, 2 n+3}
\end{array}\right] \in \mathbb{R}^{(2 n+1) \times(2 n+3)} . }
\end{aligned}
$$

Since $y(t)$ is a multi-EDS, it can be concluded that $y(t)$ is sufficiently rich of order $2 n$ for $t<+\infty$. Thanks to the linear independence of the complex vectors $\left.\left.\mathbf{G}_{1}\left(j \omega_{1}\right)\right), \cdots, \mathbf{G}_{1}\left(j \omega_{n}\right)\right)$ on the complex space $\mathbb{C}^{n}$, $\boldsymbol{\nu}_{1}(t)$ is PE for all $t \in\left[t_{\epsilon}, t_{\epsilon}+T\right]$ ([21, Chapter 2]).

Moreover, for the signal $\boldsymbol{\psi}_{2}(t)$ defined in $(27)$, there always exists a finite time interval $[\underline{t}, \bar{t}]$ with $\bar{t}>\underline{t}$ over which the elements of $\boldsymbol{\psi}_{2}(t)$ are linearly independent functions [22]. It also implies that for any $t>\underline{t}$, there exists a constant $\epsilon_{2} \in \mathbb{R}_{>0}$, such that $\int_{t-\underline{t}}^{t} \overline{\boldsymbol{\psi}}_{2}(\tau) \boldsymbol{\psi}_{2}^{\top}(\tau) d \tau \geq \epsilon_{2} \mathbf{I}$. Then, in view of $(25), \mathbf{G}_{2}$ is full row rank of $2 n+1$. Hence, we have

$$
\int_{t-\underline{t}}^{t} \boldsymbol{\nu}_{2}(\tau) \boldsymbol{\nu}_{2}^{\top}(\tau) d \tau=\mathbf{G}_{2} \int_{t-\underline{t}}^{t} \boldsymbol{\psi}_{2}(\tau) \boldsymbol{\psi}_{2}^{\top}(\tau) d \tau \mathbf{G}_{2}^{\top} \geq \underline{g}_{2}^{2} \epsilon_{2} \mathbf{I}
$$

where we denote by $\underline{g}_{2}$ the minimum singular value of $\mathbf{G}_{2}$. Note that the inequality (28) implies $\nu_{2}(t)$ PE over an interval $[\underline{t}, \bar{t}]$.

By using the fact that the sinusoidal functions in $\boldsymbol{\nu}_{1}(t)$ and the exponential functions in $\boldsymbol{\nu}_{2}(t)$ are linearly independent, it can be concluded that $\boldsymbol{\nu}(t)$ is also finite-time PE and there always exist some $\epsilon \in \mathbb{R}_{>0}, t_{\epsilon} \in \mathbb{R}_{>0}$ and $T \in \mathbb{R}_{>0}$, such that the finite-time PE condition (26) holds, thus ending the proof.

Owing to (22) and (26), it is straightforward to show that $t \in\left[t_{\epsilon}, t_{\epsilon}+T\right]$

$$
\begin{aligned}
\mathbf{R}_{f}(t) & \geq \int_{t-t_{\epsilon}}^{t} e^{-g(t-\tau)} \boldsymbol{\nu}(\tau) \boldsymbol{\nu}^{\top}(\tau) d \tau \\
& \geq e^{-g t_{\epsilon}} \int_{t-t_{\epsilon}}^{t} \boldsymbol{\nu}(\tau) \boldsymbol{\nu}^{\top}(\tau) d \tau \geq e^{-g t_{\epsilon}} \epsilon \mathbf{I},
\end{aligned}
$$


which in turn implies that, under the PE condition, the filtered auto-covariance matrix $\mathbf{R}_{f}(t)$ is invertible within a non-empty time interval $t_{\epsilon} \leq t \leq t_{\epsilon}+T$. In this connection, the unknown parameter vector $\boldsymbol{\theta}$ can be estimated by the following invertibility-conditioned estimation law:

$$
\hat{\boldsymbol{\theta}}(t)= \begin{cases}\boldsymbol{\theta}\left(t^{-}\right), & \min \operatorname{eig}\left(\mathbf{R}_{f}(t)\right)<\sigma, \\ \mathbf{R}_{f}(t)^{-1} \mathbf{S}_{f}(t), & \min \operatorname{eig}\left(\mathbf{R}_{f}(t)\right) \geq \sigma,\end{cases}
$$

where $\hat{\boldsymbol{\theta}}(0)=\boldsymbol{\theta}_{0}$ is a guessed parameter vector and $\sigma$ is an invertibility threshold set arbitrarily in the interval:

$$
0<\sigma \leq \epsilon e^{-g t_{\epsilon}} \quad \text { (noise-free scenario). }
$$

It is worth noting that the algorithm switches-off the adaptation automatically when no more excitation is detected, freezing the estimates. The invertibility of $\mathbf{R}_{f}(t)$ characterizes a sufficiently informative output signal at time t. In view of (20), the parameter $\beta$ determines the eigenvalues of $\mathbf{G}$ that in turn determines the cut-off frequency of the overall low-pass filtering structure. A larger $\beta$ results in poorer noise immunity, while a smaller $\beta$ may result in a less informative $\mathbf{R}_{f}(t)$ due to the excessive attenuation of $y(t)$. To this end, the choice of $\beta$ can be made based on a priori information of the frequencies contained in $y(t)$, such as the upper bound of all the frequency components. The tuning rules of $\beta$ also apply to $g$ (see $(23)$ ) which plays the same role as $\beta$.

As soon as the invertibility condition is met, the estimates of the coefficients $\alpha_{i}, i \in\{0, \ldots, 2 n-1\}$ and of the initial states $z_{r}(0), r \in\{0, \ldots, 2 n\}$ are immediately available. Then, in view of (4), the frequencies $\omega_{1}, \ldots, \omega_{n}$ and the damping factors $\rho_{1}, \ldots, \rho_{n}$ can be obtained respectively from the imaginary and the real parts of the roots of $P(s)$.

From (3), using the initial states $w_{i}(0)$, obtained by $\mathbf{w}(0)=\mathbf{T}^{-1} \mathbf{z}(0)$ and the frequency $\omega_{i}$, it holds that

$$
\left(\omega_{i} w_{2 i-2}(0)\right)^{2}+w_{2 i-1}(0)^{2}=A_{i}^{2} \omega_{i}^{2}, \forall i \in\{1,2, \ldots, n\}
$$

which yields

$$
A_{i}=\sqrt{\left(\left(\omega_{i} w_{2 i-2}(0)\right)^{2}+w_{2 i-1}(0)^{2}\right) / \omega_{i}^{2}} .
$$

Finally, the phase is estimated by

$$
\phi_{i}=\tan ^{-1}\left(\omega_{i} w_{2 i-2}(0) / w_{2 i-1}(0)\right),
$$

for all $i \in\{1,2, \ldots, n\}$. According to the definition in (3), the offset is obtained directly by $A_{0}=w_{2 n}(0)$.

\section{Robustness analysis in presence of bounded perturbations}

In this section, we are going to analyze the robustness of the proposed estimator in presence of a norm-bounded additive measurement noise $d(t):|d(t)| \leq \bar{d}, \forall t>0$, such that $\hat{y}(t)=y(t)+d(t)$. The following result characterises the stability properties of the estimation algorithm in the noisy scenario.

Theorem 4.1 Given the sinusoidal signal $y(t)$ and the perturbed measurement $\hat{y}(t)$, verifying the PE assumption formalized in Lemma 3.1, then the estimation error $\tilde{\boldsymbol{\theta}}(t)=\hat{\boldsymbol{\theta}}(t)-\boldsymbol{\theta}$ is ISS with respect to any disturbance signal $d(t) \in \mathcal{L}_{\infty}^{1}$.

Proof. In view of (20), performing the substitution of $y(t)$ with $\hat{y}(t)$, we get $\hat{\boldsymbol{\xi}}^{(1)}(t)=\mathbf{G}_{\xi} \hat{\boldsymbol{\xi}}(t)+\mathbf{E} \hat{y}(t)$. where $\hat{\boldsymbol{\xi}}(t)$ denotes the noisy signal in the presence $d(t)$. Defining the error variable $\tilde{\boldsymbol{\xi}}(t)=\hat{\boldsymbol{\xi}}(t)-\boldsymbol{\xi}(t)$, whose dynamic is

$$
\tilde{\boldsymbol{\xi}}^{(1)}(t)=\mathbf{G}_{\xi} \tilde{\boldsymbol{\xi}}(t)+\mathbf{E} d(t) .
$$

Thanks to the fact that the matrix $\mathbf{G}_{\xi}$ is Hurwitz, dynamic (33) is ISS with respect to $d(t)$. Each component in the vector $\tilde{\boldsymbol{\xi}}(t)$ verifies the inequalities depending on the index:

$$
\left|\tilde{\xi}_{i, j}(t)\right| \leq \frac{1-e^{-j \beta t}}{j \beta} \lambda_{i, j} \bar{d} \leq\left(\begin{array}{c}
2 n+2 \\
j-1
\end{array}\right)(j \beta)^{i-1} \bar{d} \triangleq \overline{\tilde{\xi}}_{i, j}
$$

where $i \in\{1, \ldots, 2 n+1\}, j \in\{1, \ldots, 2 n+3\}$. Thanks to the linearity of the Voterra operator, we have

$$
\left|\left[V_{K^{(i)}} d\right](t)\right| \triangleq\left|\left[V_{K^{(i)}} \hat{y}\right](t)-\left[V_{K^{(i)}} y\right](t)\right| \leq \sum_{j=1}^{2 n+3} \overline{\tilde{\xi}}_{i, j}
$$

for all $i \in\{1, \ldots, 2 n+1\}$. Then, the error signal of the regressors vector in (16) $\tilde{\boldsymbol{\nu}}(t) \triangleq \hat{\boldsymbol{\nu}}(t)-\boldsymbol{\nu}(t)$ follows that

$$
|\tilde{\boldsymbol{\nu}}(t)| \leq \sum_{i=1}^{2 n}\left|\left[V_{K^{(i)}} d\right](t)\right|=\sum_{i=1}^{2 n} \sum_{j=1}^{2 n+3} \overline{\tilde{\xi}}_{i, j}
$$

Therefore, the error-matrices $\tilde{\boldsymbol{S}}(t) \triangleq \hat{\boldsymbol{S}}(t)-\boldsymbol{S}(t)$ and $\tilde{\boldsymbol{R}}(t) \triangleq \hat{\boldsymbol{R}}(t)-\boldsymbol{R}(t)$ induced by the noise are expressed as:

$$
\begin{aligned}
& \tilde{\boldsymbol{S}}(t)=\hat{\boldsymbol{\nu}}(t)\left[V_{K^{(2 n+1)}} \hat{y}\right](t)-\boldsymbol{\nu}(t)\left[V_{K^{(2 n+1)}} y\right](t) \\
& \leq \tilde{\boldsymbol{\nu}}(t) \sum_{j=1}^{2 n+3} \xi_{2 n+1, j}(t)+\tilde{\boldsymbol{\nu}}(t) \sum_{j=1}^{2 n+3} \overline{\tilde{\xi}}_{2 n+1, j}+\boldsymbol{\nu}(t) \sum_{j=1}^{2 n+3} \overline{\tilde{\xi}}_{2 n+1, j} \\
& \tilde{\boldsymbol{R}}(t)=\hat{\boldsymbol{\nu}}(t) \hat{\boldsymbol{\nu}}^{\top}(t)-\boldsymbol{\nu}(t) \boldsymbol{\nu}^{\top}(t) \\
& =\tilde{\boldsymbol{\nu}}(t) \boldsymbol{\nu}^{\top}(t)+\boldsymbol{\nu}(t) \tilde{\boldsymbol{\nu}}^{\top}(t)+\tilde{\boldsymbol{\nu}}(t) \tilde{\boldsymbol{\nu}}^{\top}(t)
\end{aligned}
$$


which, due to the boundedness of unperturbed signals $\boldsymbol{\xi}(t)$ and $\boldsymbol{\nu}(t)$, are ISS with respect to $\tilde{\nu}(t)$ and in turn to $d(t)$ (see (34) and (35)). Eventually, the error variables of the filtered signals are bounded by

$$
\begin{aligned}
\left|\tilde{\boldsymbol{S}}_{f}(t)\right| & \triangleq\left|\hat{\boldsymbol{S}}_{f}(t)-\boldsymbol{S}_{f}(t)\right|=\int_{0}^{t} e^{-g(t-\tau)}|\tilde{\boldsymbol{S}}(\tau)| d \tau \leq \frac{1}{g}|\tilde{\boldsymbol{S}}|, \\
\left|\tilde{\boldsymbol{R}}_{f}(t)\right| & \triangleq\left|\hat{\boldsymbol{R}}_{f}(t)-\boldsymbol{R}_{f}(t)\right|=\int_{0}^{t} e^{-g(t-\tau)}|\tilde{\boldsymbol{R}}(\tau)| d \tau \leq \frac{1}{g}|\tilde{\boldsymbol{R}}|,
\end{aligned}
$$

which implies that $\left|\tilde{\boldsymbol{S}}_{f}(t)\right|$ and $\left|\tilde{\boldsymbol{R}}_{f}(t)\right|$ are ISS bounded with respect to $d(t)$.

In view of (30), in the presence of noise, it may happen either that the invertibility is not attained, thus leaving the estimate frozen at the preceding value, or for some instants the estimator is active. These two phases are separated by the user-specified invertibility threshold $\sigma$. We will show that in the latter case the mismatch between the estimate and the true parameter value is bounded and depends on the noise amplitude.

Under Lemma 3.1, in a noisy environment it holds that

$$
\begin{aligned}
|\tilde{\boldsymbol{\theta}}| & =\left|\hat{\boldsymbol{R}}_{f}(t)^{-1} \hat{\boldsymbol{S}}_{f}(t)-\boldsymbol{R}_{f}(t)^{-1} \boldsymbol{S}_{f}(t)\right| \\
& \leq\left|\hat{\boldsymbol{R}}_{f}(t)^{-1}\right|\left|\hat{\boldsymbol{S}}_{f}(t)-\hat{\boldsymbol{R}}_{f}(t) \boldsymbol{R}_{f}(t)^{-1} \boldsymbol{S}_{f}(t)\right| \\
& \leq \sigma^{-1}\left(\left|\tilde{\boldsymbol{S}}_{f}(t)\right|+\left|\tilde{\boldsymbol{R}}_{f}(t)\right||\boldsymbol{\theta}|\right)
\end{aligned}
$$

Hence, $|\tilde{\boldsymbol{\theta}}|$ is ISS with respect to $d(t)$.

Note that the estimated frequency $\hat{\omega}_{i}(t), i=1,2, \cdots$ in the presence of $d(t)$ may be 0 at some $t>0$, which results in a singularity issue in the amplitude and phase estimation (see (31) and (32)). A conservative provision is that to clip the frequency estimates by enforcing $\hat{\omega}_{i}(t)=\omega_{\min }$, where $\omega_{\min }$ is the lower bound of the input frequencies obtained based on the a priori information.

\section{Simulation Results}

\subsection{Identification of two EDS signals}

Consider the following signal

$$
y_{2}(t)=6 e^{-0.5 t} \sin \left(5 t+\frac{\pi}{2}\right)+2 e^{-0.7 t} \sin \left(2 t+\frac{\pi}{3}\right)+1 .
$$

The parameters of the kernel-based estimator are chosen as $\beta=1, g=3, \sigma=1 \times 10^{-8}$. For benchmarking purposes, the solutions are compared with the algebraic estimator developed in [11], which only estimates the damping factors and the frequencies of a two-EDS signal.
To make a fair comparison, both methods are initialized with the same guesses and are tuned to show similar convergence speed in the noise-free environment, which is verified in Figs. 1. As it can be noticed, both methods succeed in identifying the frequencies and the damping ratios with similar convergence speed in this scenario. With the same tuning parameters, we compare their behavior in the presence of a disturbance, which is assumed uniformly distributed within $[-0.2,0.2]$. It can be seen from and Fig. 2 that the algebraic method is more sensitive to the noise and is prone to diverge as the SNR decreases. In contrast, the proposed method shows an enhanced noise immunity as its estimates remain accurate in this case. In addition, the proposed method also offers precise estimates of amplitudes and initial phase angles, which are not identified by the algebraic parametric algorithm.
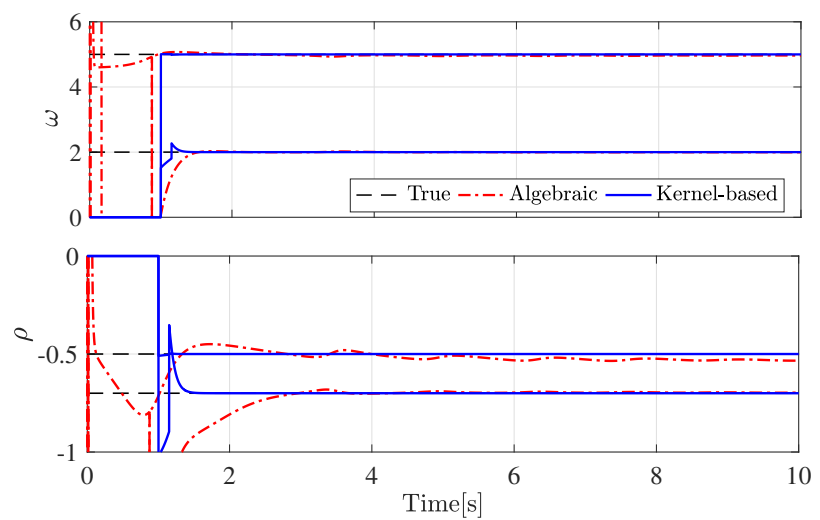

Fig. 1. Parameter estimation of two EDS signals in the noise-free scenario.

\subsection{Identification of three EDS signals}

In this example, we consider a more challenging scenario where the following measured signal is considered with two nearby frequencies:

$$
y_{3}(t)=\sum_{i=1}^{3} A_{i} e^{\rho_{i} t} \sin \left(\omega_{i} t+\phi_{i}\right)+2
$$

where $A_{1}=6, A_{2}=4, A_{3}=9, \rho_{1}=-0.5, \rho_{2}=$ $-0.7, \rho_{3}=-0.8, \omega_{1}=5 \mathrm{rad} / \mathrm{s}, \omega_{2}=3 \mathrm{rad} / \mathrm{s}, \omega_{3}=3.3$ $\mathrm{rad} / \mathrm{s}, \phi_{1}=\frac{\pi}{2}, \phi_{2}=\frac{\pi}{3}, \phi_{1}=\frac{\pi}{4}$. The estimator is tuned with: $\beta=1, g=4$ and $\sigma=1.2 \times 10^{-9}$. In the noisefree scenario (shown by the blue lines in Fig. 3), the two nearby frequencies $\left(\omega_{2}\right.$ and $\left.\omega_{3}\right)$ are precisely discriminated, while the other sinusoidal parameters are accurately identified as well. Moreover, the estimated parameters when the measurement is perturbed by a zero-mean white noise with variance $1 \times 10^{-3}$ are also depicted in Fig. 3. Despite some degradation in the accuracy of the estimates, the boundedness of all the signals in the simulation confirms the theoretical result about robustness of the algorithm in facing measurement perturbations. 

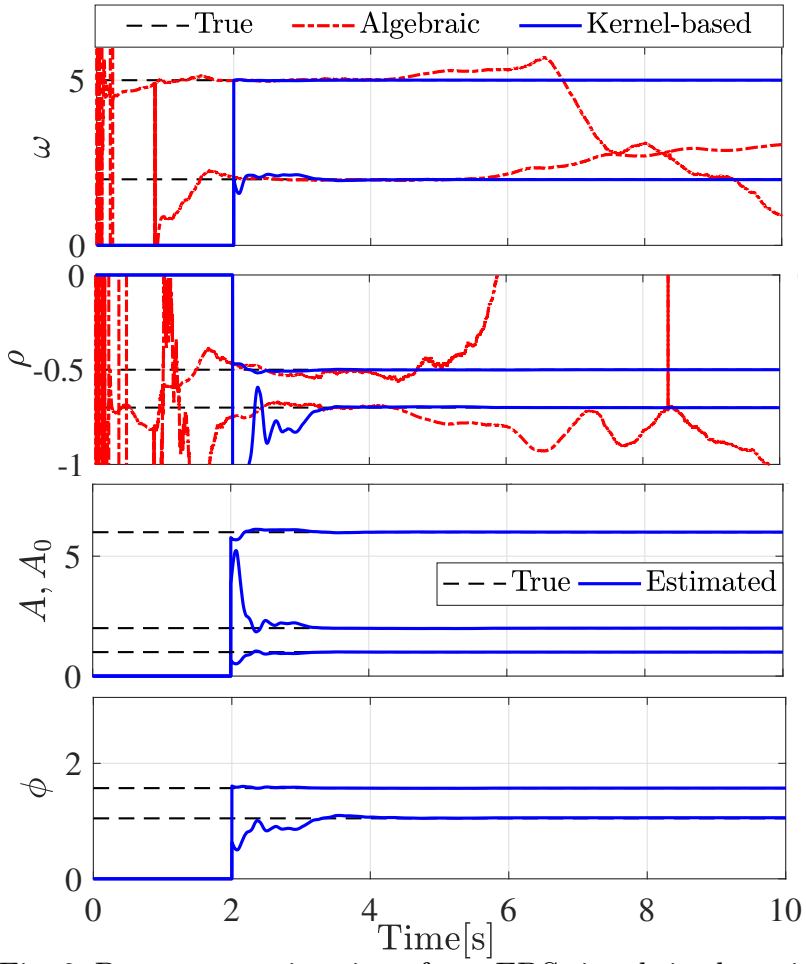

Fig. 2. Parameter estimation of two EDS signals in the noisy scenario.
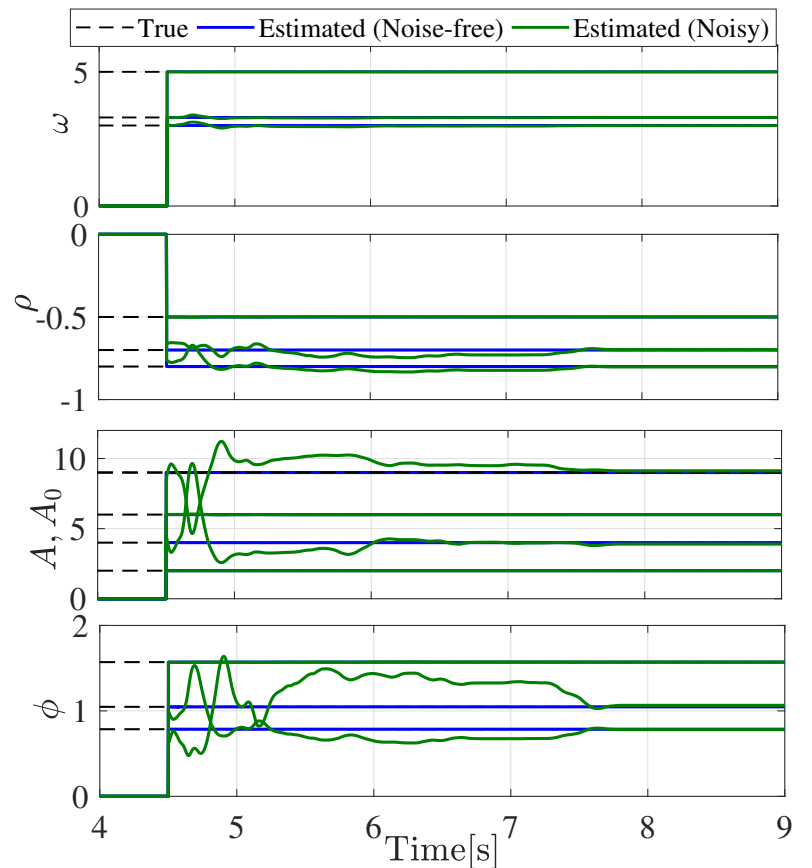

Fig. 3. Parameter estimation of three EDS signals with nearby-frequencies.

\section{Concluding Remarks}

In this paper, the problem of parametric identification of a damped multi-sinusoidal signal has been addressed. A deadbeat estimator is designed to estimate the amplitudes, frequencies and initial phase angles, damping factors and the constant bias exactly within a very short time interval. The Volterra integral operator characterized by a class of specialized kernel functions plays a key role in this framework. The proposed estimation scheme is constructed by the signals produced by this linear operator, that is implemented as an internal stable LTI system. Analytical results show that the presented methodology enjoys ISS stability properties with respect to bounded measurement perturbations. The proposed method has been thoroughly evaluated and compared with other published results by numerical examples.

\section{References}

[1] M. Osborne and G. K. Smyth, "A modified Prony algorithm for fitting functions defined by difference equations," SIAM Journal on Scientific and Statistical Computing, vol. 12, no. 2, pp. 362-382, 1991.

[2] M. Osborne and G. K. Smyth, "A modified prony algorithm for exponential function fitting," SIAM Journal on Scientific Computing, vol. 16, no. 1, pp. 119-138, 1995.

[3] R. W. Wies, J. W. Pierre, and D. J. Trudnowski, "Use of ARMA block processing for estimating stationary lowfrequency electromechanical modes of power systems," IEEE Transactions on Power Systems, vol. 18, no. 1, pp. 167-173, 2003.

[4] R. Badeau, R. Boyer, and B. David, "EDS parametric modeling and tracking of audio signals," in Proc. of the 5th International Conference on Digital Audio Effects (DAFx), 2002, pp. 139-144.

[5] K. Shin and J. Hammond, Fundamentals of signal processing for sound and vibration engineers. John Wiley \& Sons, 2008.

[6] B. Wu and M. Bodson, "A magnitude/phase-locked loop approach to parameter estimation of periodic signals," IEEE Trans. on Automatic Control, vol. 48, no. 4, pp. 612-618, 2003.

[7] G. Pin, "A direct approach for the frequency-adaptive feedforward cancellation of harmonic disturbances," IEEE Trans. on Signal Processing, vol. 58, no. 7, pp. 3513-3530, 2010.

[8] B. Chen, G. Pin, W. M. Ng, C. K. Lee, S. Y. R. Hui, and T. Parisini, "An adaptive observer-based switched methodology for the identification of a perturbed sinusoidal signal: Theory and experiments," IEEE Trans. on Signal Processing, vol. 62, no. 24, pp. 6355-6365, 2014.

[9] A. A. Bobtsov, D. Efimov, A. A. Pyrkin, and A. Zolghadri, "Switched algorithm for frequency estimation with noise rejection," IEEE Trans. on Automatic Control, vol. 57, no. 9, pp. 2400-2404, 2012.

[10] G. Fedele and A. Ferrise, "Non adaptive second order generalized integrator for identification of a biased sinusoidal signal," IEEE Trans. on Automatic Control, vol. 57, no. 7, pp. 1838-1842, 2012.

[11] A. Neves, M. Miranda, and M. Mboup, "Algebraic parameter estimation of damped exponentials," in Proc. EUSIPCO, 2007, pp. 965-969.

[12] M. Mboup, "Parameter estimation for signals described by differential equations," Applicable Analysis, vol. 88, no. 1, pp. 29-52, 2009.

[13] J. Lu and L. J. Brown, "Identification of exponentially damped sinusoidal signals," in Proc. of the 17th IFAC World Congress, 2008. 
[14] M. Mansouri, M. Mojiri, M. A. Ghadiri-Modarres, and M. Karimi-Ghartemani, "Estimation of electromechanical oscillations from phasor measurements using secondorder generalized integrator," IEEE Transactions on Instrumentation and Measurement, vol. 64, no. 4, pp. 943950, 2015.

[15] G. Pin, A. Assalone, M. Lovera, and T. Parisini, "Non-asymptotic kernel-based parametric estimation of continuous-time linear systems," IEEE Trans. on Automatic Control, vol. 61, no. 2, pp. 360-373, 2016.

[16] G. Pin, B. Chen, and T. Parisini, "Robust finite-time estimation of biased sinusoidal signals: A Volterra operators approach," Automatica, vol. 77, pp. 120-132, 2017.

[17] P. Li, G. Fedele, G. Pin, and T. Parisini, "Kernel-based deadbeat parametric estimation of bias-affected damped sinusoidal signals," in Proc. of the 2016 European Control Conference (ECC), Aalborg, DK, pp. 519-524, 2016.

[18] B. Chen, P. Li, G. Pin, and T. Parisini, "Estimation of multisinusoidal signals: A deadbeat methodology," in Proc. of the IEEE Conference on Decision and Control, Las Vegas, USA, 2016, pp. 3763-3768.

[19] T. Burton, Volterra Integral and Differential Equations. Elsevier, 2005.

[20] G. Pin, M. Lovera, A. Assalone, and T. Parisini, "Kernelbased non-asymptotic state estimation for linear continuoustime system," in Proc. of the IEEE American Control Conference, Washington, DC, 2013, pp. 3123 - 3128.

[21] S. Sastry and M. Bodson, Adaptive Control: Stability, Convergence, and Robustness. Prentice-Hall, 1994.

[22] G. Sansone, Orthogonal Functions: Revised English Edition. Dover Publications, 1991.

[23] M. Mboup, "Parameter Estimation via Differential Algebra and Operational Calculus," INRIA Research Report, 2007. 